\title{
Reflections on Routley's Ultralogic Program
}

\author{
Daniel Nolan \\ Department of Philosophy \\ University of Notre Dame
}

It is a common thought that logic is "topic neutral", at least in the sense that no matter what the subject of reasoning, logic should be a fit tool for tackling it. One way of spelling out this idea is offered in a papercum-manifesto published by Richard Routley ${ }^{1}$ in 1977, called "Ultralogic as Universal", which is best known in its reprinted form as an appendix to Routley 1980a. (Page references will be to that appendix.) Routley argued that, given the job of logic, the kind of logic we should adopt is a relatively prooftheoretically weak relevant logic. While the paper was in part a discussion of future avenues for logical investigation, Routley offered specific answers to a range of foundational logical questions.

It is safe to say that Routley's manifesto was not widely taken up, and indeed the wider relevance/relevant logic tradition of which he was a part is on the fringe of work on logic in philosophy departments as a whole these days. However, the desiderata he points to should be of interest to philosophical logicians whether or not they see themselves in Routley's tradition, and the arguments he gives for the features of his preferred logics should be addressed by relevant and non-relevant logicians alike: those who do not wish to follow his lead should articulate why. At any rate, since I feel the pull of some of Routley's desiderata, or at least something like them, I think it is worth examining Routley's criteria to think about what sorts of approaches might satisfy them.

In this paper, I will take up three tasks in turn. The first is to set out what Routley thought we should demand of an all-purpose universal logic, and some of his reasons for those demands. The second is to sketch Routley's

\footnotetext{
${ }^{1}$ In 1983 Routley changed his name to Richard Sylvan. In this paper I will refer to him as Routley when discussing his views about ultralogic which he published under that name. I should note that it is not clear whether he continued to hold these views: in Sylvan 1997, he adopts a pluralism about logic, though it seems that his preferred "plurallism" may still provide a privileged place for the sort of logic that he formerly defended as universal. I will not attempt to relate his earlier views to his 1997 position here, however.
} 
own response to those demands. The third is to explore how else we could satisfy some of the theoretical demands Routley identified, if we are not to follow him in endorsing Routleyan Ultralogic as a foundational logic. As part of this third project, I want to articulate what seems to me a preferable way of going to respond to the challenges Routley correctly identifies: and while I doubt what I will have to say would have convinced Routley himself, I will try to show that the approach I prefer has several advantages over Routley's.

\section{The Tasks of a Universal Logic}

Routley accepted (Routley 1980a p 986) that there were some special-purpose logics for particular purposes. But he also thought there was one logic that was privileged: that it was universal, and that it was the logic best suited for "foundational studies" in metalogic, mathematics, linguistics and elsewhere. Indeed, according to Routley, the best way to understand more local logics is to see them as relying on deductive reasoning that could be made explicit through reasoning in the universal logic with additional premises known (or believed) to be true for the case at hand: much as intuitionist logicians have sometimes suggested that classical logic can be recaptured by adding as additional premises all instances of excluded middle.

This underlying logic was intended to apply wherever deductive reasoning could be applied: he sought a logic that is "applicable in every situation whether realised or not, possible or not... including illogical, inconsistent and paradoxical ones" (Routley 1980a p 893). Many logics have adherents willing to claim that they are the "one true logic", but Routley criticised many of the other obvious alternatives (such as systems incorporating classical logic or intuitionistic logic) on the grounds that those logics failed for many "deductive reasoning situations". These include circumstances where we are faced with inconsistent or incomplete bodies of information, reasoning about paradoxes such as the Liar, and reasoning about the belief systems of nonideal agents. Some of Routley's criticisms of other universal projects such as classical logic will be discussed further below.

Routley did not simply assert that we should aim to have a universal logic, so characterised. He offers a range of arguments for thinking that adopting a universal logic is preferable, and against arguments that it is not feasible (Routley 1980a pp 896-898). One kind of reason consists in drawing attention to weaknesses of alternatives, particularly drawbacks of the view that all logics are local. For example, one problem for the idea that there is no universal logic, but that instead there are only subject-matter specific logics, is that this sort of localism "runs into difficulties at... boundaries". It 
struggles to explain how to do reasoning that involves premises from different subject matters, or how to choose which logic to use when it is unclear what area some information falls into.

Routley also deals with objections to the idea of a universal logic, including the idea that for any logic we can find a sufficiently illogical situation where it will break down. One might initially think that Routley's demand is that we find a universal logic that governs our reasoning whenever we reason, or at least whenever we deductively reason (setting aside inductive reasoning, arguments from analogy, and other obviously ampliative cases). ${ }^{2}$ On closer inspection, however, it turns out that the "deductive reasoning situations" may be a narrower class of circumstances to reason about than those. Routley admits that "situations can always be found which fail to conform to any specific logical principles" (Routley 1980a p 898). He does not there give specific examples, but some cases which I think Routley would agree are cases of this sort include reasoning about what someone in fact believes, given that they believe a set of claims $A_{1}$ through $A_{n}$ : even if $B$ is a fairly uncontroversial consequence of $A_{1}$ through $A_{n}$, actual believers do not always draw such inferences. Or for an even clearer case, just because someone has explicitly said $A_{1}$ through $A_{n}$, it does not follow that they have explicitly said $B$, no matter how secure a logical consequence $B$ is of $A_{1}$ through $A_{n}$. The "situation" of the content of our believer's beliefs or our asserter's utterances are situations we cannot suppose are closed under logical consequence.

When we engage in deductive reasoning that involves these situations, Routley's preferred way to make this reasoning explicit seems to be to cast it in terms of formulating the reasoning using "highly intensional functors" (p 898). So, for example, if I have a situation not closed under conjunction elimination (an example Routley talks about elsewhere, when discussing a fiction in which principles of connexive logic govern (Routley 1979 p 11-12)), I will be able to make claims of the form "according to the fiction $(A \& \sim A)$ ", while rejecting the corresponding claim "according to the fiction $A$ "without myself violating conjunction elimination: since when I represent "according to the fiction" with an operator $O, O(A \& \sim A)$ need not have as a logical consequence $O(A)$, even if $A \& \sim A$ has $A$ as a consequence.

It is worth addressing an ambiguity to be resolved in understanding Routley's talk of "reasoning situations". In one sense of "reasoning situation", the situation in question is one where the reasoner finds herself: with various beliefs or pieces of evidence, subject to various cognitive and environmen-

\footnotetext{
${ }^{2}$ Routley himself may have thought we can recapture most or all apparently "inductive" reasoning e.g. in science in a deductive framework: See e.g. Routley 1980a pp 946-949. But let us leave such cases aside for the sake of a tractable discussion.
} 
tal constraints, and so on. In the other sense of "reasoning situation" the situation in question is one in which the premises obtain - it may be a mathematical situation, a situation in the distant past, an inconsistent situation, a situation in fiction, etc. It is clear from context that the reasoning situations Routley is concerned with are the second sort. He is concerned about the range of topics or bodies of information logic should be applied to. This talk can sound rather platonistic: why should there be a fictional situation, just because there is a story, or a 'belief situation' just because there are beliefs? For those with such scruples, I suspect this talk of situations can be paraphrased away, in this discussion at least, by talking about collections of propositions or bodies of information instead. I am inclined to commitment to possible and impossible worlds and situations myself, so I think some good sense can be made of talk of e.g. counterfactual situations or even impossible, inconsistent, or paradoxical situations. So I will follow Routley in helping myself to the "situation" locution, including the slightly loose talk of deduction or reasoning or a logic being about situations. (Even though I think there are situations, I do not think that inferences are typically literally about situations, as opposed to what the premises or conclusions are more naturally taken to be about.)

The second requirement on a successful logic is that it be a "paradox solvent". A lot of work in philosophical logic in the last hundred years has focused on constructing logics that satisfactorily handle standing puzzles, and many proposed revisions to e.g. classical logic have been motivated on the basis that they provide more satisfactory solutions to these puzzles. (Among the "paradoxes" Routley thought it was important to address were the "paradoxes of material implication" and the "paradoxes of strict implication" that motivate many conditional logics.) Note that to be a "paradox solvent" a logic need not avoid all the conclusions of traditional paradoxes that theorists have found distasteful. Routley, for example, advocated solutions to the Liar paradox that treated the Liar sentence as being both true and false (and indeed treated the truth and falsehood of the Liar sentence as theorems of a suitable truth theory). He also defended the doctrine that some things do not exist, which many have found an intolerable way out of the puzzles about thought and talk of the non-existent. Being a "paradox solvent" is presumably a matter of both being able to represent and derive entailments from paradoxical sentences, and perhaps to provide the materials to resolve paradoxes, or at least to do so when the paradox is one that arises in logic or related areas. (If we include as "paradoxes" every case where plausible premises lead to apparently unacceptable conclusions, it would be odd to suppose that logic should provide the solution to all paradoxes.)

The third requirement is that the logic be sensitive to very fine-grained 
distinctions in the meanings of expressions. For example, it should not treat as logically equivalent expressions which share a referent, or an extension: for that matter, it should not treat as logically equivalent expressions that necessarily co-designate. Furthermore, in Routley's preferred logics, sentences which logically entail each other are not intersubstitutable in all contexts salva veritate. In the terminology introduced by Cresswell 1975, Routley's preferred logics are sensitive to hyperintensional distinctions between expressions. Routley himself preferred the expression "ultramodal" for logics sensitive to these distinctions, and his label "ultralogic" for his preferred logic stems from its sensitivity to ultramodal distinctions (Routley 1980a p 894). (The term "logically equivalent statements" is sometimes used to mean expressions that are logically interderivable from each other, and sometimes used to mean that they can be always substituted in any context salva veritate. I will use the term sense of mutual interderivability in this paper, since when it comes to fine-grained enough logical systems, the only expression always substitutable salva veritate with a sentence $s$ is $s$ itself.)

A terminological aside: I am not sure why Routley preferred the expression "ultramodal", but I have three conjectures. When the expression "hyperintensional" was first introduced, it was used both for contexts in which logical equivalents could not be substituted salva veritate, but quickly also came to apply to contexts where necessary equivalents could not be substituted salva veritate. This ambiguity led to few problems in practice, but Routley may have thought an expression that unambiguously picked out the latter would be desirable. The second is that it avoids an awkwardness in the classifications "extensional", "intensional" and "hyperintensional": as they are normally defined hyperintensional constructions are a subspecies of intensional constructions, which leaves us no entirely convenient way of saying that a particular construction is intensional while not being hyperintensional ("merely intensional"). Routley seems to have used "modal" to pick out the intermediate category of non-extensional constructions that are not ultramodal (see p $894 \mathrm{n} \mathrm{0}$ ), thus more clearly delineating the three categories of interest. The third is based on an observation of Max Cresswell's (personal communication): "hyperintension" commits the etymological sin of mixing Greek and Latin roots, while "ultramodal" is a more respectable Latinism. Whatever the reason for Routley's terminological suggestion, "ultramodal" has not become a mainstream alternative to "hyperintensional".

There are a number of areas where we would not want to be able to substitute necessarily equivalent expressions unrestrictedly. Propositional attitude contexts are one well known case: knowing one mathematical truth does not mean one knows every other mathematical truth equivalent to it, and there is more than one way to believe something impossible. If Kripke 
is right, "it is a priori that..." does not allow unrestricted substitutions of necessary equivalents, since some necessary truths are a priori truths but others are not. And so on for a growing list of cases. (See Nolan 2014 for a recent partial list.)

Of particular significance in this context is the fact that Routley also thought it was important to be able to represent in a logic facts about entailment: i.e. facts about what followed from what. An arrow formula that is meant to represent entailment should not allow for substitution of necessary equivalents, since necessary equivalents will have different logical consequences. Presumably logical axioms are all meant to be true and indeed necessarily true: but in axiomatic logics with more than one, non-redundant, axiom, one axiom will not be an entailment of any of the others, even though it will typically be an entailment of itself. So a device to represent entailment relationships should be "ultramodal". It is particularly desirable to be able to represent entailment relations in a logic intended to be universal, since a universal logic must be able to represent deductions about its own features, on pain of logic being silent about those deductive relationships altogether.

\section{Routley's Proposal For Meeting Those De- mands}

Routley had a specific proposal for what form this universal, all-purpose logic should take. Three aspects of this proposal stand out. It should be relevant: that is, it should fall in the family of "relevant" or "relevance" logics explored in e.g. Routley et al. 1983. (Routley 1980a pp 898-900) It should be "dialectical": that is, it should accept the existence of true contradictions (pp 916-919). (This is a feature that Routley and Graham Priest would later label dialetheist.) It should be ultramodal (p 894), not treating necessarily equivalent expressions as logically equivalent (e.g. in a logic of belief, it should not follow from someone's believing one necessary truth that she believes any other arbitrary necessary truth. So even when A and $B$ express necessary truths, we cannot freely substitute them to go from "Ann believes A" to "Ann believes B"). Another condition that Routley would no doubt impose on an ultralogic is that it would have "neutral" quantification, quantifying over objects that do not exist as well as those that do: and while this is the central theme of Exploring Meinong's Jungle, where "Ultralogic is Universal" appears as an appendix, it does not come in for discussion in the latter work, so I will leave it aside for the current discussion. 
Characterising what it is for a logic to be a "relevant" or "relevance" logic is not entirely easy or uncontroversial. One popular characterisation of the propositional relevant logics is that all of the theorem arrow-formulas of the language "share variables": no propositional variable in the consequents of the theorems fails to appear in the antecedent. Routley himself favoured a characterisation in terms of "sufficiency" of antecedents to consequents in entailments ( $\mathrm{p}$ 895-6), though this characterisation (and the suggestion that other logics do not have premises sufficient for their conclusions without supplementation with other information) is obviously tendentious. When explaining the superiority of relevant logics as candidates to be a universal logic, Routley leaned heavily on the advantages of being paracomplete and paraconsistent: offering the logical possibility of truth-value gaps and truthvalue gluts.

The sentential logic that is at the core of Routley's proposed logic for mathematics, at least, is one he calls DK (p 918), to which he adds quantificational principles, principles for identity, principles governing set membership, and other devices. DK is a weak relevant logic: it is significantly weaker than $\mathrm{R}$, the relevant logic Routley (along with a number of others) is most associated with, since DK lacks contraction. (It is similar to, but slightly weaker than, the "dialectical logic" of Routley and Meyer 1976). As I read him, he would think DK is still too strong to be the sentential fragment of his ultimate ultralogic: he notes that doing justice to incompleteness requires more weakening of its axioms (p 919). ${ }^{3}$ Routley does not explicitly endorse a candidate ultralogic in the paper, so far as I can determine. I imagine his preferred option would lie somewhere between the strength of DK, just mentioned, and a very weak relevant logic like B (see Routley et al. 1983). ${ }^{4}$ For applications, of course, additional premises or premise schemas will often be needed, and much of Routley 1980a involves sketching how we might recover the desirable parts of e.g. classical mathematics with underlying logics much weaker than classical logic.

In arguing that a universal logic should be "dialectical", Routley intends two things. One is that it is paraconsistent: that it should not enable the derivation of arbitrary formulas from a contradiction. The other is that the

\footnotetext{
${ }^{3}$ Ross Brady has suggested to me that despite the misgiving mentioned here, Routley might still have preferred DK for an ultralogic on balance (personal communication).

${ }^{4}$ Thanks to discussion with Ross Brady and David Ripley here, who convinced me that Routley would likely want a stronger sentential basis than B. One possibility is that he would have endorsed the logic DJ, as discussed e.g. in Brady 1996. Thanks to discussion with Ross Brady and Zach Weber about which logical systems Routley would have endorsed for an ultralogic. My own view is that he was undecided on the best option at the time of writing.
} 
logic should be dialethic: it should admit the existence of contradictions that are in fact true. (In some dialethic logics including a truth predicate, including ones Routley favoured, some contradictions are even logical theorems.)

An important commonly mentioned motive for this is for a logic that will satisfactorily handle the semantic and set-theoretic paradoxes, such as the Liar, paradoxes of designation such as Grelling and Nelson's "heterological" paradox, and set theoretic paradoxes like Russell's or Burali-Forti's. If we just accept that Liar sentences are both true and false, and both true-andnot-true, accept that "heterological" both is and is not heterological, that the Russell set both belongs to itself and does not, we can preserve relatively naive theories of truth, designation, membership, and so on. But another motive Routley mentions that bears specifically on a logic's pretensions to universality is our ability to reason non-trivially about rival solutions to paradoxes like these. We want to be able to consider solutions which postulate inconsistency or incompleteness hypothetically without our hypothetical reasoning being trivial or otherwise collapsing, and according to Routley non-paraconsistent logics "simply rule out proper logical examinations of incomplete and inconsistent deductive theories, in particular of non-vacuous incomplete theories where not all logical laws hold, and of non-trivial inconsistent theories where some contradictory propositions hold" (Routley 1980a p 898). Even before we decide whether these theories are correct, we must be able to investigate them and reason about situations in which they obtain. A logic that throws up its hands when exploring these theories does not have a wide enough range of application, or so Routley argues.

Finally, it should not be a surprise that if we specify that one of the jobs of a universal logic is to enable us to deductively reason in a way that we can distinguish necessary equivalents and logical equivalents, then a logic that will adequately fit that bill will itself be "ultramodal" or hyperintensional. We have already seen above some of the reasons to want a logic that can handle hyperintensional constructions. Routley was keen on logics of belief that allow us to distinguish necessarily equivalent beliefs; logics of significance which do not run together necessarily equivalent but non-synonymous expressions; counterfactuals which are not trivially true when their antecedents are impossible, and so on. At the time of writing Routley 1980a he projected writing a book with Valerie Plumwood (then Valerie Routley) with the title Beyond the Possible (p 961 note 29): that title sums up a significant portion of Routley's logical work. ${ }^{5}$

\footnotetext{
${ }^{5}$ Zach Weber tells me that the footnote cited of Routley's might have been superseded by events by 1980, and that the projected "Beyond the Possible" work was just the singleauthored Exploring Meinong's Jungle itself.
} 


\section{Alternatives to Routley's Proposal}

While few have endorsed a one true logic along the lines of Routley's Ultralogic, each one of the desiderata he outlines (universality, being a paradox solvent, and being ultramodal/hyperintensional) are of wide appeal. The demand for a universal logic has been traditionally popular, and for good reasons. For one thing, it has seemed to many that logic is a universal part of method: that when premises and conclusions are in the right logical form, they stand in the corresponding deductive relationships, and logic does not suddenly break down when applied to some areas of human inquiry rather than others. It has a track record as a go-anywhere science. For another, the meanings of logical particles such as "and" or "if" do not seem to be wildly ambiguous: if there are important commonalities whenever they are used, these include some commonalities in the inferential behaviour of sentences they occur in.

A final reason to look for a universal logic has been touched on above. We need to be able to reason about the relationships between different subject areas, and if we lacked a logic that applied across different topics, it is very hard to see how we could do this. That's not yet an argument that a universal logic is to be had: perhaps it just turns out that we are cosmically unlucky and there is no logic-like way to reason across domains of inquiry. But at least insofar as we think we can and do reason using premises from all manner of subject matters to conclusions in other subject matters (perhaps via "mixed" conditional claims or other "mixed" compound expressions), we have a reason to think there is some logical structure underlying the information we have across domains.

The demand for a logic that is a "paradox solvent", or is at least paradoxresistant, is also hard to resist. After all, if we endorse a logic that leads to incorrect conclusions from correct premises, we are stuck with being incorrect. Furthermore, the apparent consequences of some paradoxes are especially absurd. Endorsing a trivial theory which entails every proposition, or endorsing every claim employing vague language that is the conclusion of some sorites argument, would be to indulge in intolerable absurdity. If we are to use logic when talking of truth, or employing vague expressions, or any other area where paradoxes dwell, we do not want limitations of the logic to lead us astray. I doubt we should demand that logic itself resolve the problem whenever we have apparently acceptable premises leading to an apparently unacceptable conclusion: sometimes we should just reject one of the premises or be led to embrace the conclusion. So insisting that our logical theory be the source of solutions to all paradoxes would be too extreme. But at the very least we should demand that our logical systems and the theory 
associated with them avoid, defuse or diagnose logical paradoxes. It is hard to see where else, besides our broadly logical inquiries, we should be looking to resolve them.

Finally, our deductive reasoning had better be capable of drawing hyperintensional distinctions. As discussed above, there are many different important constructions that do not permit substitution of necessary equivalents: and if we are to understand their roles in deductive inferences, it seems we must admit hyperintensionality into our logical formalism as well.

Routley offers one kind of approach that holds out the promise of a universal, paradox-free, ultramodal/hyperintensional logic. In this section I will look briefly at three rival ways we could try to secure the logical benefits Routley seeks without employing his methods. It may be that these three alternatives are not exhaustive, though they seem to me to cover most of the available options.

\subsection{Traditional Universal Logics}

An alternative that would act as a head-on response to Routley's criteria would be to offer a rival logic that was universal, came with a suite of solutions to paradoxes, and which made enough discriminations to handle reasoning about all manner of apparently hyperintensional matters. There are many potential ways to do this. One conservative way would be to argue that much more restricted resources were needed to handle every reasoning situation. One conservative approach would be to insist that classical logic, suitably extended, is all the logic we need, and that standard modal resources are enough to understand all the reasoning we need to do, even when hyperintensional resources are apparently called for.

Ordinary unvarnished classical logic does not allow for unrestricted substitution of necessary equivalents: even if "2 is a number" or "water is $\mathrm{H}_{2} \mathrm{O}$ " are necessary truths, their straightforward formalisations are not interderivable. Modal logics weaker, particularly those weaker than S5, do not allow for arbitrary substitution of formulas even when we add as premises that those formulas are necessary: in many models of $\mathrm{T}$, for example, some necessary formulas are possibly not-necessary, while others are necessarily necessary. So when Routley complains that logics like this are not "ultramodal", he presumably has something else in mind besides the demand that logics should not allow unrestricted substitution of necessary equivalents. Two aspects of his complaint are that many logics treat contradictions as uniformly false (and, in modal logics, necessarily false), and that the philosophy around the meanings of philosophically interesting expressions was dominated by approaches which conflated impossibilities. 
Logics that extend classical logic, intuitionistic logic, or straightforward logics of truth-value gaps such as strong Kleene logic are all in Routley's sights. Formulating an inconsistent theory, e.g. of the Liar or naive set theory, and closing it under any of these three logics produces triviality, since in all of these any conclusion can be validly deduced from a formal contradiction. They do not appear to offer good frameworks for working out better or worse ways of developing inconsistent theories, since they treat all formally inconsistent theories as equivalent and trivial. They also struggle to represent failures of excluded middle: even though intuitionism and strong Kleene both have model theories with truth value gaps and do not have excluded middle as theorems, attempting to state that there is a failure of excluded middle in either logic is not straightforward. $(\sim(A \vee \sim A)$ is just equivalent to $(\sim A \& \sim \sim A)$ in both. $)$ Not even being able to sensibly reason using inconsistent or some incomplete theories is one mark against them, in Routley's book.

It is possible to resist the need for any hyperintensional operators in an overall adequate theory, and the meaningfulness of hyperintensional operators is called into question by some influential traditions in philosophy. After the eclipse of extensionalism (which was far from dead when Routley was writing in 1977), perhaps the main systematic challenge to making sense of hyperintensionality has come from the possible worlds semantics championed by figures such as Richard Montague, David Lewis, and Robert Stalnaker. According to this approach meanings of different expressions can be built up as functions from possible objects and possible worlds: names and definite descriptions as functions from worlds to objects, ordinary predicates as functions from worlds to extensions in those worlds, sentences as functions from worlds to truth values, or alternatives sets of worlds, and so on. According to the most straightforward versions of possible worlds semantics, the meanings of necessarily co-referring names are identical, the meanings of necessarily co-applying predicates are identical, and the meanings of necessarily equivalent sentences are identical. If a language is compositional, distinctions between necessary equivalents get collapsed: if Robin Hood and Robin of Locksley are necessarily the same, then "The sheriff seeks Robin Hood" and "The sheriff seeks Robin of Locksley" will be synonymous. Once we treat constructions like "Beth believes that..." as sentential operators, Beth will believe the logical equivalents of everything she believes. Who knew discovering mathematical identities was so easy? Impossible propositions all make the same contribution to truth values of constructions which have them as constituents, as well. So, for example, any counterfactual containing an impossible antecedent will have the same truth value as a counterfactual with the same consequent and different impossible antecedent, at least if the coun- 
terfactual construction is treated as operating on propositions expressed by antecedents and consequents.

Defenders of possible worlds semantics have tried a variety of techniques to keep to the letter of their view while trying to preserve the appearance of hyperintensionality. One is to adapt a manoeuvre Quine and Davidson employed when defending extensionalism and rely on the distinction between use and mention. Stalnaker, for example, suggests that we attribute belief or knowledge of some mathematical truths but not others to people despite there only being one necessarily true mathematical proposition, because attribution of mathematical beliefs (and perhaps many other beliefs) relies for their truth not just on what the proposition is that would be expressed by the expression following "believes that", and what propositions the person believes, but also on facts about the relationship between mathematical sentences and the person (Stalnaker 1984 pp 73-75). In some perhaps extended sense, these attributions are about the expressions displayed as well as what they mean.

This device can also be used to discuss the apparent hyperintensionality of resources used in a theory of logic itself. Not everything formally entails an arbitrary necessary truth. Does it follow that "entails" (or "logically entails") is hyperintensional? Not automatically. If "entails" is not a propositional operator, but a predicate that expresses a relation between sentences, or logical forms, or somesuch, then " $2+2=4$ entails $2+2=4$ " need not mean the same as " $2+2=4$ entails water is $\mathrm{H}_{2} \mathrm{O}$ ", since the latter talks about the sentence "water is $\mathrm{H}_{2} \mathrm{O}$ " (or some structure akin to the sentence). Or at least this is a natural move to make if one holds, on theoretical grounds, that "water is $\mathrm{H}_{2} \mathrm{O}$ " and " $2+2=4$ " share the same meaning.

Here is not the place to engage in a sustained critique of these approaches. The idea that we cannot make sense of impossibilities or reason cogently about them is not initially appealing, though enough years of training can presumably make it look natural or even unavoidable. The devices for interpreting belief as only about possibility, or being somehow metalinguistic, or entailment claims as surreptitiously mentioning sentences, also seem procrustean. In principle, it may be worth living with these limitations for a theory that is simple enough, or explanatory enough, or otherwise has weighty considerations in its favour. Whether or not the possibilities-only paradigm is sufficiently ahead of alternatives is something to be decided on the basis of examining how it does vis a vis its rivals across a range of specific areas. ${ }^{6}$ My own view is that it is less satisfactory than taking hyperinten-

\footnotetext{
${ }^{6}$ I discuss the prospects of extensionalist and possible-worlds-semantics approaches to apparently hyperintensional phenomena in more detail in Nolan 2014.
} 
sionality more at face value, and allowing for greater generosity about our ability to reasonably manage our thought and claims about impossibilities. But I doubt there is much that can be said quickly to dislodge theorists who wish to stick to their guns. Let us then look at two alternatives to this traditional approach of sticking with a strong logic and avoiding hyperintensional operators in our theory of deductive reasoning.

\subsection{Pluralism}

Another alternative is give up the universal pretensions of logic. We could do this by deeming some topics permanently off-limits for logical investigation, but a less radical approach would be to adopt a logical pluralism, according to which for every reasoning situation there is at least one correct logic for that situation, but there is no useful logic correct for every reasoning situation. Instead of a single universal logic, a pluralist can offer a patchwork of different principles for different occasions. (No doubt there are other motivations for logical pluralism, and logical pluralists need not think that logics are topicspecific in this way: but I am interested here in appealing to logical pluralism as an alternative to adopting a universal logic, as opposed to other reasons one might wish to endorse pluralism. ${ }^{7}$ ) Note the negative claim, that there is no go-anywhere logic, is important to pluralism as I am construing it here (though others may of course use the word "pluralism" differently.) Even a believer in a universal logic may agree that there is a plurality of correct "logics" for different reasoning situations which differ from the universal logic, if we can recover those logics by adding some premises to a universal logic or otherwise treating local logics as special cases of the universal one.

Pluralists are likely to resist the demand that the logic we use for "foundational" investigations be its own meta-logic, at least if this is a general demand: the topic of logic itself might well be different from the topics suitable for other logics! Avoiding "logical closure" is also a way to avoid a range of paradoxes of self-reference. Pluralists cannot avoid these paradoxes altogether, at least if they accept that there are some language/logic combinations that can talk about themselves, or talk about truth in general, etc., but at least pluralists can hope to take a more divide-and-conquer approach if they are not constrained to always allow logics are their own meta-logics. Pluralists also have open to them, as a general approach, allowing that dif-

\footnotetext{
${ }^{7}$ The kinds of logical pluralism I am exploring here can be contrasted with the kind of logical pluralism defended by Beall and Restall 2006: their pluralism gives us a family of logics that can be used with any premises whatever, but deliver different results and are supposed to be fit for different purposes. The pluralisms I am interested in yield collections of logics each of which should only be applied to some subject matters.
} 
ferent systems can have different features we would like our theory-plus-logic to have, and may hope to defuse paradoxes by conceding that different logics have different features we want, even if those features are never found together in a legitimate logic. Pluralists are unlikely to object to allowing that at least some logical systems are ultramodal, even if they wish to also allow that some logics are e.g. entirely extensional. So pluralists have some options that seem to be denied to the universalist.

Pluralists have a lot of room to move in dealing with the problems we want a theory of logic to solve. And there are enough potential pluralist options that I would be foolhardy to try to evaluate them all here, let alone to even try to show that they are all unsatisfactory compared to adopting a universal logic. But there are a few general drawbacks that pluralism has that make a search for a universal logic worthwhile, it seems to me. For one thing, as Routley noted, there are puzzles about how to understand all our deductive reasoning together, especially if conclusions in one area bear on questions in another. For another, it is likely that a pluralistic theory of logic will be less unified and less simple than its universalist rivals. Instead of a single logic (supplemented by additional premises when necessary), a pluralist will have several or perhaps many different logical systems which correctly describe reasoning in different areas, plus a superstructure of theory saying which system properly applies where, together with the appropriate explanations of why, and how to manage inferences that "mix" premises or sub-arguments from different areas. A unified and simpler theory of valid inference should be preferred, everything else being equal. ${ }^{8}$

Finally, pluralism seems initially implausible. Treating logic as describing unrestricted validity seems to have enjoyed some success over the history of the subject, and it is difficult to see in the case of particular inferences which are candidates for validities in one case how they could fail to be valid elsewhere due to a change of subject matter. How could $A \& B$ be true without $A$ ?

A closely related option would be to embrace "logical nihilism" and deny that either universal logic or plural, local logics are successful in their job description. If taken as an entirely general claim, this would be a difficult position to establish, given the pedestrian purposes logic is sometimes put to: surely some bodies of information are regimented in a way so that e.g. they are closed under conjunction? If so, using some ordinary logical principles when dealing with that information seems an innocuous enough way to pass from truths to other truths. ${ }^{9}$ An interesting position in the vicinity would be

\footnotetext{
${ }^{8}$ I note that Routley also approved of making the choice between logical theories on the basis of theoretical virtues: see Routley 1980b.

${ }^{9}$ Though for a sympathetic discussion of such a general logical nihilism, see Russell
} 
some form of local logical nihilism: not denying that all logics are everywhere inapplicable, but rather than there are some subject matters unfit for logic. These might be the content of fiction, or the content of mental states, or theology, or ethics, or the deliverances of mystical experience, or whatever. If some domains escape the applicability of logic, that could relieve us of the dilemma of either searching for principles that governed those domains as well as others, or specifying a local logic of that subject and how it related to local logics of other domains. I don't myself think that either global logical nihilism or even this partial logical nihilism are very appealing options compared to rival approaches, in the end, so I will not stop to develop the option more here.

None of this is intended to be a knock-down refutation of pluralism (or of nihilism for that matter). Pluralism in particular remains a live alternative to the quest for a single go-anywhere logic, and potentially avoids some of the challenges the postulation of a universal logic faces.

\subsection{Reasoning in the Scope of Ultramodal Operators}

The final alternative I see to an Ultralogic along the lines of Routley's proposal is to seek a universal logic, but to understand many of our "reasoning situations" in a different way from Routley. It is to treat a lot of our reasoning as implicitly from premises prefixed by ultramodal operators to conclusions also prefixed by those operators. In effect, it is to treat much more of our reasoning, even logical-looking reasoning, as involving "non-deductive situations" in Routley's sense of that expression. There are well behaved situations, such as those captured by the actual truth and other situations which are logically possible scenarios. But then there are also other situations we want to reason about, including impossible fictions, impossible theories, alternative logical hypotheses, semantic hypotheses, situations with alternative mathematics, and so on. We want to allow that we can make sense of alternative logics, or "illogical" belief systems, or surreal fictional worlds, while at the same time keeping our logical moorings. And I think this is feasible while staying monistic about logic: one system of deductive validity can be recognised, while not treating everything we can sensibly reason about as closed under our favourite logic.

The way to do this is to treat a lot of our reasoning about the "irrational", "illogical", "paradoxical" and so on as implicitly in the scope of hyperintensional operators. In particular, I think we should carefully distinguish deduction in the scope of a conditional from deduction outside the scope of a

2017.

Australasian Journal of Logic (15:2) 2018 Article no. 3.8 
conditional. Suppose I want to reason informatively about an inconsistent scenario-for example, one where naive set theory is correct-even though I think logic rules out inconsistencies. A straightforward option would be to list some starting points as premises, and use logic to derive conclusions. (This is, I take it, what Routley advocates, though he holds of course that we should adopt a logic that does not rule out all inconsistencies.) Another way is to reason from some conditionals to others. "If naive set theory is true, then the Russell set is a member of itself and not a member of itself", therefore "If naive set theory is true, then there is at least one inconsistent set", for example. However, concluding from the premise "If naive set theory is true, all sets are members of themselves" would be a bad piece of reasoning, when we are trying to consider naive set theory sympathetically. To put it in the (optional) language of reasoning situations, we can reason about inconsistent reasoning situations without using premises or conclusions that are themselves inconsistent: and we can do so without going metalinguistic either. (The antecedents in this example are about the truth of a theory, but more cumbersome antecedents which are just the conjunctions of an axiomatisation of naive set theory would do as well.) Of course, to do this, we need a hyperintensional understanding of the relevant conditionals, so we cannot substitute necessary equivalents (or even logical equivalents) willy-nilly into antecedents or consequents.

Once we allow ourselves to reason in this conditional fashion about impossibilities, we can extend ourselves even further, and treat reasoning under a supposition, or hypothetical reasoning, as functioning the same way as reasoning in the scope of a conditional. In Nolan 1997 pp 555-559 I suggest that we can model hypothetical reasoning as reasoning in the scope of counterfactual conditionals. To parallel the above case, imagine I reasoned by saying "suppose naive set theory were true. Then the Russell set is a member of itself and not a member of itself. So at least one set is a member of itself and not a member of itself". One way to understand what has happened is that the effect of the reasoning is much the same as before: I have not categorically supported the conclusion that at least one set is a member of itself and not a member of itself: instead, the force of my conclusion is captured by "if naive set theory were true, at least one set is a member of itself and not a member of itself". Exactly what the connection between this sort of supposition and counterfactual conditionals is could be a subject of some further dispute: do we merely model reasoning employing a supposition with counterfactuals? Or are the counterfactuals the logical form of suppositional reasoning? Or something else? Whatever exactly it is, if it has the same logical strength as the corresponding counterfactuals, it does not require us to expand our logic so that naive set theory is genuinely logically possible: it merely ob- 
tains in situations we can reason about conditionally, or suppositionally, or hypothetically.

One advantage this approach has over the proposal to count e.g. naive set theory as holding in a non-trivial situation closed under a universal logic, is that this account generalises better. Routley allows naive set theory is non-trivial and logically possible, but he holds that other logical and mathematical proposals violate his ultralogic. If we want to reason about what the situations where it can be that $A \& \sim A$ but fail to be that $A$, we cannot find non-trivial situations closed under Routley's preferred logics like that. But we can apply the strategy I propose of reasoning conditionally or suppositionally about such hypotheses: and given appropriate background, it would be correct to reject the transition from "If ( $A$ and not- $A$ ) is the case but conjunction elimination fails for the case of ( $A$ and not- $A$ ), then $A$ and not- $A$ " to "If ( $A$ and not- $A$ ) is the case but conjunction elimination fails for the case of ( $A$ and not- $A$ ), then $A$ ". Routley can of course take situations that are not closed under conjunction elimination to be "non-deductive", and perhaps even reason about them conditionally: but it seems he does not have a unified account of different apparently counter-logical cases, since he expands his logic to allow for the possibility of one case but must treat the other in a very different way.

You might worry that this conditionals-heavy approach to reasoning with theories sketched above throws the baby out with the bathwater: have I been left with too little for categorical, non-hypothetical, logic to do? I think not, for two reasons. One job for non-hypothetical logic to do is to tell us which transitions from which are genuinely truth-preserving (in virtue of logical form, etc. etc.), and this seems to me a topic of great logical interest whatever else we might investigate. The second is that as well as hypothetical reasoning, we also employ categorical reasoning: we reason from premises we take to be in fact correct, in an attempt to reach conclusions that are in fact correct. When we seek sound deductive reasoning, and not merely valid transitions, it is often because we are after conclusions that are correct and fit for belief. Non-hypothetical reasoning is the standard we should be responsive to when attempting to reason soundly from premises we take to be true.

We can keep some proof-theoretically strong principles in our logic and nevertheless have devices for representing reasoning about logically strange situations, provided we have conditionals that tolerate logically strange antecedents and consequents without misbehaving or going trivial. Conditionals and suppositional or hypothetical reasoning are among the most powerful, but others may have a role in particular cases. When using deductive methods about what a reasoner believes, for example, we could take some of their 
belief contents as premises and deduce propositions from them that we also attribute as beliefs. That will often make sense in practice: if someone standing in front of a house believes that if there is no barking, there is no dog inside, and they believe there is no barking, it is normally safe to predict that they believe there is no dog inside as well. But it is unlikely to be an exceptionless method: people's beliefs are not always, or perhaps ever, closed under logical consequence in general. Instead, it is better to take as premises e.g. "Anna believes there is no barking" and "Anna believes that if there is no barking, there is no dog inside", plus ancillary principles of psychology and facts about Anna, to yield the prediction "Anna believes there is no dog inside". In general, many interesting operators will not have their contents closed under logical consequence: and for many of them, it it is implausible that they are closed under logical consequence even if the logic is as weak as Routley's ultralogic.

My guess is that Routley would complain that a logic of the sort I prefer, relying heavily on hyperintensional operators, would not be general enough, since it would not cover every "deductive reasoning situation". As we have seen above, Routley himself concedes that his preferred ultralogic will not cover every situation about which we might want to reason: he admits there are interesting "non-deductive reasoning situations" as well as deductive ones. So whether the option sketched in this section is general enough will likely turn on the question of when we are allowed to think that a "situation" of interest is not closed under logical consequence, and when we have to accept that it is. One place where such a disagreement might play out concerns the "situations" we consider in counterfactual reasoning. The approach I favour does not insist that these are closed under any interesting logic, and this will be reflected by the behaviour of transitioning between counterfactuals which share an antecedent: very few inferences of the form $A \rightarrow B$, therefore $A \rightarrow C$ will be formally valid. (Perhaps none besides the case where $B$ is the same as $C$ : see Nolan $1997 \mathrm{p}$ 547-554). If all the situations relevant to counterfactual evaluations were closed under an "ultralogic" (at least one that went beyond the identity inference), then one would expect that inferences of the form $A \rightarrow B$ therefore $A \rightarrow C$ would be valid whenever $C$ was a logical consequence of $B$.

Ironically, the approach I prefer is the approach Routley himself adopted towards the "reasoning situations" created by discussion of fiction. One situation we often face is taking some claims we hold are true according to a fiction, and reasoning from them to others that we conclude are true according to a fiction. (Sherlock Holmes lives in London, Watson lives in London, therefore Holmes and Watson live in the same place, for example.) Given this phenomenon, you might initially expect Routley to think that our ul- 
tralogic should not find any counterexamples among the transitions we make when reasoning about fictional matters, even when dealing with fictions that we might traditionally have thought were "badly behaved" logically. After all, ultralogic should not have counterexamples in any deductive reasoning situation, whether or not that reasoning involves literal truths.

Instead, Routley denies that fictions, in general, are closed under any interesting logical operations (Routley 1979 p 10-11). He does think that there will be a set of logical principles that can be used when dealing with "usual works of fiction" ( $\mathrm{p}$ 11), but in full generality there is very little that follows as a matter of logic about what else is true according to a fiction, given a claim that is true according to a fiction. (Formally, if we let $O$ be an operator for "it is true according to the fiction that", from $O(A)$ almost nothing follows as a matter of logic about whether $O(B)$, where $B$ is not the same formula as $A$.) "[T]here is no general uniform logic of fiction", Routley says (Routley 1979 p 10, italics Routley's). In particular, Routley argues that the principle $O(A \& B) \rightarrow O(A)$ will fail, in full generality (p 11). The reason Routley gives is that authors can create stories that violate any usual principles: his counterexample for $O(A \& B) \rightarrow O(A)$ is a story where connexive logics are correct (and they allow for counterexamples to the entailment of $A$ from $A \& B$, while Routley's preferred ultralogical options treat that transition as valid).

I agree with Routley about this aspect of the logic of fictions: fictions can be arbitrarily strange and logically anarchic. I am tempted to treat a lot of not-explicitly-prefixed reasoning about fictional matters as implicitly prefixed by something like an "according to the fiction" operator, and allow that very little of that reasoning, so understood, is logically valid as opposed to nondeductively strong in virtue of background information we have about how the particular fiction at hand works. But Routley's willingness to treat fictional situations as not universally closed under an ultralogic leaves Routley in a slightly awkward dialectical situation. He demands that all "deductive reasoning situations" be closed under logic, but has no obvious criteria for which apparently deductive reasoning situations are genuinely "deductive reasoning situations" and which are "non-deductive" apart from the apparently circular one of checking to see whether the situation is closed under logic. When fiction appears to provide counterexamples to his ultralogic, it turns out some fictional situations we reason about are "non-deductive" after all.

At present, I do not see a way to motivate the distinction Routley wants between deductive and non-deductive reasoning situations that draws the line where he appears to want to draw it. (Routley assures us that the class of deductive situations is "independently and naturally determined" (Routley 
1980a p 898), but does not say much more about how, except by reference to which situations are closed under the correct ultralogic.) Without such a motivation, there does not seem to be any cogent objection to treating a wide range of puzzle cases the way Routley wants to treat fiction: as not being in general closed under any interesting logic, and playing a role in our reasoning via reasoning implicitly prefixed by hyperintensional operators.

There is another, more serious concern about the approach of treating the logic of hypothetical reasoning in the way I suggest that Routley would also no doubt raise. For a logic to be paradox-free, it should at least not take us from true premises to logically unacceptable conclusions. Even if we are equipped with a system that allows us, for example, to reason about a story containing a naive truth theory without treating that story as trivial, when it comes time to deal with the Liar paradox we cannot just accept all the premises of an argument that the Liar is both true and false, together with principles that would allow us to in turn infer unacceptable consequences from this, or even infer triviality from a contradiction. So even after we have helped ourselves to a wide range of ultramodal operators with various exotic properties, we must still return to the traditional stock of logical paradoxes and explain how we can maintain appealing logical principles while avoiding absurd consequences. The fact that we can handle logically unusual situations in hypothetical reasoning without compromising cherished logical principles would be no help if it were shown that there are actual counterexamples to those principles.

I do not have an all-purpose solvent for logical paradoxes to hand, and I admit that even after we have a satisfactory counterpossible conditional of the sort I have indicated, serious questions remain about the rest of the logical resources we might accept. The correct solution to semantic paradoxes, or paradoxes of vagueness, or other paradoxes where the problem might be logical, may be to endorse a logic that lacks many of the inferences or theorems that are classically valid. If avoiding paradoxes required a logic for non-hypothetical reasoning that could be applied as-is to a sufficiently wide range of reasoning with impossible premises and in drawing out consequences of those premises, then that would be an important argument for that approach over a logic that was not greatly revised in light of the paradoxes and and handles exotic cases using an apparatus of hyperintensional operators (e.g. counterfactuals to represent hypothetical reasoning). So my preferred approach holds out a hostage to fortune in aiming for solutions to a range of paradoxes that are not too logically revisionary.

Note that introducing new hyperintensional operators is likely to raise new logical paradoxes as well, either due to those operators by themselves or the interaction between them and resources already in our formal languages. 
Examples include Fitch's knowability paradox, Kaplan's cardinality paradox for combining modal and intentional operators in general (Kaplan 1995), Löb's paradox for provability predicates, and so on. I have argued that a suitable hyperintensional conditional can avoid the associated Curry paradox (Nolan 2016), but other operators that we would want in our language, such as a sentential connective to signal validity of inference, will bring with them Curry paradoxes that are hard to solve (see e.g. Beall and Murzi 2013).

A final concern is whether the principles governing what inferences can be drawn within the scope of these hyperintensional operators should count as part of "logic" at all. Some conceptions of logic see it as general and domainneutral: but the distinctive principles a "logic of belief" concerns only one narrow part of human psychology, for example. The principles governing an "according to fiction $X$ " operator seem to have even narrower application, and so on. To some extent this is perhaps a matter of terminology: similar issues were raised about whether modal logic is "logic", given its relatively restricted subject matter. What we have are interesting connections between formal, syntactically specifiable, properties of a language and what inferences are truth-preserving. Whether we want to call that system a "logic" or not, we would like to have a good theory of the formally specifiable correct rules of inference for use with a "believes that" construction, or a "true according to" construction, or for that matter sentential operators corresponding to "necessarily" or "possibly". Whether we treat the resulting theories as part of "logic" proper or part of an inquiry in the philosophy of language may not matter very much for anything other than institutional and sub-disciplineboundary-policing purposes.

A different concern about the "logical" status of hyperintensional operators ("believes that", "says that", "grounds", etc.) is that there may not be very many interesting formal principles that govern inferences they crucially contribute to. Given that A believes one thing, there may not be much else we can logically deduce about what else A believes, for example, though there may be psychological regularities to be discovered. Likewise, even if there are general regularities about what grounds what, or what explains what, these might best be seen as no business of logic. The concern about whether formally stateable principles governing these operators are genuinely "parts of logic" may end up being terminological: just as a debate about whether the axioms of arithmetic are, or are not, part of "logic" can be rather sterile in the abstract. The concern that there will be few formally stateable inferences that are truth-preserving and which crucially rely on these operators is less likely to be a mere terminological concern. I suspect with some popular hyperintensional operators, there are not many distinctive, formally characterised inferences that involve them: "according 
to the fiction...", "believes that...", or even "it is obligatory that..." may not license many distinctive inferences which are formally stateable, suitably general, and counterexample-less. It still seems to me worth debating how these operators contribute to valid deductive inference, and whether there are interesting truths of the sort one usually expects from a logic: but it may turn out that the "logic" of these operators is very weak, contrary to what is assumed by many who write on "doxastic logic" or "the logic of fiction" or "deontic logic", and so on. But in my book very weak logics are logics too.

\section{Conclusion}

Coming up with a single go-anywhere logic remains an appealing goal, and ensuring that it does not fall to logical paradoxes and does not conflate necessary equivalents are both hard to disagree with. However, I think the best way forward towards those goals looks very different from Routley's. We need to be able to deductively reason non-trivially and successfully even about hypothetical situations where principles we might ordinarily accept have broken down. Likewise, and leaving aside the controversial talk of situations, we need to be able to make non-trivial sense of bodies of information that are not closed under logical principles that we favour. But this is not best done by weakening our logic until those bodies of information are closed under the logic we end up endorsing.

Instead, when we want to reason when engaged with these bodies of information we should employ "ultramodal" or hyperintensional operators: and we should be prepared to analyse reasoning where these operators are not on the surface as implicitly involving them. In the case of hypothetical reasoning about apparently logically impossible theories, this can be done by reinterpreting the reasoning as being in the scope of conditionals: instead of deductions from one part of the theory to consequences of it, it is instead reasoning from premises about what would be the case if the theory were true to conclusions concerning what would be the case if the theory were true. In the case of reasoning about the goings-on in impossible fictions, we can reconstruct the reasoning as taking us from claims of the form "according to the fiction, P" to claims of a similar form, yielding other claims about what is true according to the fiction. The need to explore apparently impossible or illogical alternatives is no reason to weaken our logic pre-emptively.

We have no guarantee that there will be a universal canon of deductive validity, though it seems to me reasonable to hope that one will be available, and to adopt that aim in our logical theorising, if only for the pragmatic reason that if we find we cannot have such a universal canon the reason for 
our failure will point the way to what to try instead. We should not try to find a logic that will apply directly to every "reasoning situation", however: instead, we should expect that there are many cases where reasoning should be reconstructed as implicitly happening in the scope of hyperintensional operators. This general suggestion needs to be fleshed out further: though my discussion of hypothetical reasoning and the case of "according to the fiction" operators can stand as two examples. We should expect of a logic with universal pretensions that it can be a standard for all valid deductive reasoning that occurs, even when we consider hypotheticals, or fictions, or rival solutions to paradoxes, or other contents that take us well beyond the logically possible. ${ }^{10}$

\section{References}

Beall, Jc and Restall, G. 2006. Logical Pluralism. Oxford: Oxford University Press

Beall, Jc and Murzi, J. 2013. "Two Flavors of Curry's Paradox". Journal of Philosophy 110.3: 143-165

Brady, R. 1996. "Relevant Implication and the Case for a Weaker Logic". Journal of Philosophical Logic 25.2: 151-183

Cresswell, M. J. 1975. "Hyperintensional Logic". Studia Logica 34.1: 25-38

Kaplan, D. 1995. "A Problem in Possible Worlds Semantics" in SinnottArmstrong, W., Raffman, D. and Asher, N. (eds) Modality, Morality and Belief: Essays in Honor of Ruth Barcan Marcus. Cambridge: Cambridge University Press, pp 41-52

Nolan, D. 1997. "Impossible Worlds: A Modest Approach". Notre Dame Journal for Formal Logic 38.4: 535-572

Nolan, D. 2014. "Hyperintensional Metaphysics". Philosophical Studies 171: 149-160

Nolan, D. 2016. "Conditionals and Curry". Philosophical Studies 173.10: 2629-2647

Routley, R. 1977. "Ultralogic as Universal?" Relevance Logic Newsletter 2.12: $50-90$ and 138-75

\footnotetext{
${ }^{10}$ Thanks David Ripley, Ross Brady, Chris Daly, Dominic Hyde, Zach Weber and to audiences at the 2016 ASL conference in Storrs and the 2016 AAL conference in Melbourne for valuable feedback and advice.
} 
Routley, R. 1979. "The Semantical Structure of Fictional Discourse". Poetics 8: $3-30$

Routley, R. 1980a. Exploring Meinong's Jungle. Canberra: Research School of Social Sciences, Australian National University

Routley, R. 1980b. "The Choice of Logical Foundations: Non-Classical Choices and the Ultralogical Choice". Studia Logica 39.1: 77-98

Routley, R. and Meyer, R.K. 1976. "Dialectical Logic, Classical Logic, and the Consistency of the World". Studies in Soviet Thought 16: 1-25

Routley, R., Meyer, R.K., Plumwood, V. and Brady, R. 1983. Relevant Logics and its Rivals. Vol. 1. Atascadero: Ridgeview

Russell, G. 2017. "An Introduction to Logical Nihilism" in Leitgeb, H., Niniluoto, I. Seppala, P. and Sober, E. (eds) Logic, Methodology and Philosophy of Science - Proceedings of the 15th International Congress. London: College Publications, pp 125-134

Stalnaker, R. 1984. Inquiry. Cambridge: MIT Press

Sylvan, R. 1997. Transcendental Metaphysics: From Radical to Deep Plurallism. Winwick: White Horse Press 